Albrecht Greule, Sandra Reimann, Anna Schuster

\title{
FikTive FEINDBILDER UND VERSCHLEIERTE GEWALT SprachWissenschaftliche ANALysen ZUM INTERVIEW MIT EINEM ISLAMISTEN ${ }^{1}$
}

\section{Sprache als Mittel der Identifikation}

Die umfassende Reichweite des Begriffs Sprache wird einem bereits beim Versuch einer allgemeinen Definition vor Augen geführt und dehnt sich bei einer differenzierteren Betrachtung zunehmend aus. Allein im Digitalen Wörterbuch der deutschen Sprache etwa stößt man auf vier verschiedene Bedeutungen, die sich zwar ähneln, aber dennoch gegenseitig ergänzen und somit nur in ihrer Gesamtheit einer einheitlichen Begriffserklärung annähern. Dabei bezeichnen die letzten drei Eingrenzungen die Fähigkeit und die Art des Sprechens bzw. den Akt des Sprechens an sich, während eine erste auf ein „Instrument des begrifflichen Denkens [einer] bestimmten Einheit der menschlichen Gesellschaft" 2 verweist. Jene Erläuterung geht über eine rein funktionale Dimension des sprachlichen Austauschs zur Vermittlung von Inhalten hinaus und verweist ebenso auf eine repräsentative Wirkung von Sprache, die innerhalb einer Gemeinschaft entsteht und diese nach außen hin positioniert und abgrenzt. Sprache ist somit ein vereinfachendes Zeichensystem einerseits, das neben außersprachlichen Mitteln wie Gestik und Mimik überhaupt erst die zwischenmenschliche Kommunikation ermöglicht, und ein abstraktes System andererseits, durch dessen individuellen Gebrauch sich persönliche Haltungen abzeichnen.

„Übernehmen Sie die Worte ihres Gegenübers, übernehmen Sie auch seine Sichtweise" (Ryborz 2012: 97) - mit diesen Worten beschreibt Heinz Ryborz in einem rhetorischen Ratgeber das von Sprache bestimmte Miteinander. Damit wird deutlich, dass Worte eben nicht nur aus lexikalischen

\footnotetext{
1 Vorliegender Beitrag ist eine überarbeite Version der Bachelor-Arbeit von Anna Schuster. Vorgelegt bei der Philologischen Fakultät III der Universität Regensburg am 30.09.2015.

2 Vgl. www.dwds.de/wb/Sprache (12.02.2019).
} 
Einheiten bestehen, sondern darüber hinaus trotz ihres neutralen Denotats Einstellungen ausdrücken können, die auf eine weitere Bedeutungsebene hinweisen. Diese Ebene wird greifbarer, wenn man sich Ferdinand de Saussures Auffassung von Sprache als ein System aus sprachlichen Zeichen vor Augen führt, wonach jene Zeichen nichts anderes als eine Verbindung aus dessen Vorstellung und dessen Lautbild sind. Diese basiert zugleich auf Beliebigkeit und Konventionalität, d. h., die Motivation der Verbindung ist zwar beliebig, deren Gebrauch jedoch konventionell. Das Zeichen beruht demnach wie ,jedes in einer Gesellschaft rezipierte Ausdrucksmittel im Grunde auf einer Kollektivgewohnheit, oder, was auf dasselbe hinauskommt, auf der Konvention" (De Saussure 1967: 80). Der Begriff der Konvention verweist auf den sozialen Charakter von Sprache, der im Sinne der langue - des sprachlichen Zeichensystems als theoretisches Konstrukt - unabhängig vom Individuum ist. Im Sinne der parole - der konkreten Realisierung und Anwendung jenes Systems - ist diese Konvention zwar nach wie vor gegeben, gleichzeitig wird jedoch der Bezug des Individuums zur Sprache ersichtlich (vgl. De Saussure 1967: 17f.). Hinsichtlich derjenigen sprachlichen Zeichen, die nicht auf greifbare Objekte zurückzuführen sind, bleibt die zu einem Lautbild gehörende Vorstellung letztlich ein geistiges Konstrukt, das ein Sprecher während jeglicher Art der Kommunikation auch beim Rezipienten in identischer Form vorzufinden glaubt. Ob die Vorstellung eines sprachlichen Zeichens, das auf einen abstrakten Sachverhalt Bezug nimmt - als Beispiel wäre etwa Toleranz zu nennen - bei verschiedenen Sprechern identisch ist, ergibt sich letzten Endes jedoch nur aus dem Kontext, in dem dieses verwendet wird.

Mithilfe eines spezifischen Sprachgebrauchs also kann ein Sprecher eine bestimmte Einstellung ausdrücken, ohne diese als vollständige, inhaltliche Aussage zu formulieren. Aus diesem Grund bilden sich letztlich Sprachmuster durch gesellschaftliche Gruppen bzw. Gruppen durch Sprachmuster. Im Folgenden wird der Sprachgebrauch eines IS-Anhängers $^{3}$ in Bezug auf dessen Gruppenzugehörigkeit und die damit verbundene Einstellung untersucht.

Einer dieser Anhänger ist der deutschsprachige Islamist Erhan A. ${ }^{4}$, der sich im von Marie Delhaes und Frederik Obermaier durchgeführten Interview des SZ-Magazins offiziell zum Islamischen Staat bekennt. Die allge-

${ }^{3}$ Die Abkürzung IS bezeichnet im Folgenden die terroristische Vereinigung Islamischer Staat.

${ }^{4}$ Im SZ-Interview, das als Primärquelle vorliegt, wird kein vollständiger Name des Interviewten angegeben. 
Fiktive Feindbilder und verschleierte Gewalt. Sprachwissenschaftliche... 41

meine Fragestellung, die diesem thematisch zugrunde liegt, formulieren die Autoren im Vorfeld des eigentlichen Interviews: „Was bringt junge Menschen, die hier aufgewachsen sind, dazu, sich einer radikalen Terrormiliz zuzuwenden?" (Delhaes/Obermaier 2014: 13).

Jenes Interview soll im Folgenden als Korpus dienen, um mögliche islamistische Denkmuster anhand von konkreten, sprachlichen Äußerungen $\mathrm{zu}$ erfassen und zu deuten.

\section{Bedeutungsebenen des Radikalen und Extremen}

\subsection{Zur Definition von Radikalismus und Extremismus}

Der Gebrauch von Sprache verweist im Zuge der eigenen Identitätsfindung und Positionierung innerhalb der Gesellschaft noch lange nicht auf darin enthaltene radikale oder gar extreme ${ }^{5}$ Denkmuster. Um festzustellen, wann Sprache nicht lediglich den Zweck eines gemeinschaftlichen Orientierungssystems erfüllt, sondern darüber hinaus einen radikalen oder extremen Charakter annimmt, bedarf es zunächst einer Klärung der beiden Begriffe. Dabei soll der Fokus gerade deshalb auf Radikalismus und Extremismus - und nicht etwa auf andere politische Termini wie Terrorismus - gelegt werden, da sich daraus spezifische Sprachmuster ableiten lassen, die eine sprachwissenschaftliche Untersuchung überhaupt erst ermöglichen. Im Vordergrund steht schließlich nicht, wie Gruppierungen wie der Islamische Staat politisch einzuordnen sind, sondern welche konkreten Denkmuster sich aus deren kollektiven Sprachgebrauch entnehmen lassen. Dennoch sollte an dieser Stelle darauf verwiesen werden, dass die Erfassung jener Muster eine gewisse „disziplinäre[r] Mobilität“ (Fuest/Löffler 2005: 11) erfordert, da selbst die sprachwissenschaftliche Beleuchtung erst unter Berücksichtigung des gesamten Diskurses zu einem schlüssigen Ergebnis führen kann. So schließt die Analyse einer gruppenspezifischen Verwendung von Sprache stets auch die individuellen, sozialen Umstände sowie die gesamte, gesellschaftliche Situation, mit der sich eine Gruppe konfrontiert sieht, ein. Aus diesem Grund erscheint es sinnvoll, ein Phänomen wie die Sprache des Radikalismus und Extremismus zwar als Forschungsgegenstand der Linguistik zu analysieren, sich diesem aber dennoch unter Einbezug des Gesamtzusammenhangs anzunähern.

\footnotetext{
${ }^{5}$ Da nicht die politische Form des Extremismus im Vordergrund steht, sondern lediglich dessen Charakter, wird im Folgenden das Wort extrem anstatt extremistisch verwendet.
} 
Bevor also entsprechende Sprachmuster veranschaulicht werden, um diese schließlich zu erkennen und zu deuten, sollen beide Begriffe zunächst in ihrer philosophischen und politischen Bedeutung erfasst werden. Bezüglich ihres namentlichen Ursprungs bilden sie eine Art Gegensatz, der nur auf den ersten Blick widersprüchlich erscheint und bei genauerer Betrachtung eine gegenseitige Definierbarkeit ermöglicht. So ist Radikalismus auf das lateinische radix, die Wurzel, und Extremismus auf extremum, das Äußerste, zurückzuführen - d. h., zunächst stehen sich Inneres und Äußeres als konträre Topologien gegenüber (vgl. Willer 2005: 61). Da eine genaue Abgrenzung nach wie vor umstritten ist, kann die Erläuterung nicht einfach auf Basis allgemeingültiger Definitionen fortgeführt werden, weshalb die weitere Eingrenzung nun in erster Linie auf jener wörtlichen Deutung fundiert ist. Demnach kann bereits eine innere Überzeugung, die sich sozusagen aus der Wurzel einer Fragestellung ergibt, als radikal bezeichnet werden, während sich deren gegebenenfalls „extremer Charakter [...] erst in Extremsituationen" (Kurbacher 2005: 54) offenbart, in denen jene Überzeugung bis zum Äußersten getrieben wird. Radikalismus muss demnach nicht negativ gedeutet werden und kann sich lediglich auf die individuelle Haltung beziehen, während Extremismus eine „homogene[n] Gemeinschaft" (Bötticher/Mareš 2012: 58) anstrebt und damit selbsttätig die Unterdrückung derjenigen zur Folge hat, die die entsprechende Haltung nicht teilen. Damit wird die wechselseitige Beziehung beider Begriffe deutlich: Das Beharren auf einer solchen Überzeugung ist radikal, während deren zwanghafte, gewaltsame Verbreitung innerhalb einer Gemeinschaft extrem ist. Reduziert auf die gegenseitige Relation lässt sich also sagen: „Alle Extremisten sind radikal, aber [...] Radikale sind keine Extremisten“" (Bötticher/Mareš 2012: 54).

\subsection{Radikale und extreme Sprache}

Die Terrormiliz Islamischer Staat ist - wie jede terroristische Vereinigung - radikal in ihrer Weltauffassung und extrem in ihrem Handeln: Ihre offizielle Hymne verspricht den Anhängern „den Sieg der wahrhaft Gläubigen" (Said 2014: 65) und damit die Errichtung des Kalifats, des islamischen Gottesstaats. Allein diese wenigen Worte reichen aus, um die Versprachlichung beider Phänomene zu erahnen. Wird von Sieg und wahrhaft Gläubigen gesprochen, muss anderswo von Niederlage und Ungläubigen ausgegangen werden. 
Fiktive Feindbilder und verschleierte Gewalt. Sprachwissenschaftliche... 43

Nun hat der Versuch einer Definition jedoch verdeutlicht, dass Radikalismus - als Ursprung des Extremismus - zunächst einmal aus einer individuellen, inneren Überzeugung heraus entsteht, d. h., der bedingungslose Glaube an den Aufbau eines Kalifats und dessen Konsequenzen ist nicht von Anfang an innerhalb einer Gemeinschaft verwurzelt, sondern impliziert durch dessen Verbreitung überhaupt erst die Entstehung einer solchen Gemeinschaft. Die radikale Überzeugung an sich ist also zunächst ein „innersubjektives Phänomen“ (Kurbacher 2005: 50), das durch den „Vorgang des Überzeugens [zum] intersubjektive[n] Phänomen" (Kurbacher 2005: 50) wird. Hier nun setzt die Sprachgebundenheit des Radikalismus an: Um eine Überzeugung nach außen zu transportieren und damit jenen intersubjektiven Vorgang auszulösen, bedarf es deren sprachlicher Inszenierung.

Wie also äußert sich radikale und im Falle des Islamischen Staats extreme Sprache? Zur Radikalisierung kann es nur dann kommen, wenn eine Überzeugung bezüglich einer Fragestellung als „richtige“ und einzige Lösung angenommen wird, d. h. wenn die Vielfalt von Weltanschauungen - die stets auf ein Subjekt zurückzuführen und damit nicht objektivierbar sind - auf einen engen Ausschnitt reduziert wird (vgl. Schlosser 1995: 139). Jener Ausschnitt kann somit nicht mehr einer umfassenden Realität entsprechen, da sämtliche Umstände, die der Überzeugung nicht Genüge tun, ausgeblendet werden. Entsprechend dieser gedanklichen Verengung muss sich letztlich also auch die Vielfalt der Sprache reduzieren. Diese wird somit zum Zwecke der gedanklichen Manipulation eingesetzt, indem sie selbst manipuliert wird. Radikalismus ist demnach schlichtweg die „Verweigerung von Sprache“ (Schlosser 1995: 139).

\section{Das Interview mit Erhan A. als Korpus}

\subsection{Erhan A. als Beispiel einer Zielgruppe}

Olaf Lang verweist bei einer Abhandlung über manipulative Sprache darauf, dass jene nicht ausschließlich auf bestimmte Klassen oder Gruppen einer Gesellschaft abzielt (vgl. Lang 1991: 52). Letztlich ist niemand aufgrund seiner Zugehörigkeit zu einer als vermeintlich „weniger anfällig“ eingeschätzten Gesellschaftsschicht vor der Beeinflussung durch jene Sprachmuster bewahrt, d. h., gesellschaftliche Kategorien wie das Bildungsniveau oder der finanzielle Status sagen nicht im Vornherein etwas über die grundsätzliche Empfänglichkeit einer Person gegenüber den genannten, sprachlichen Mitteln aus. 
Dennoch begünstigen bestimmte, individuelle Faktoren die Beeinflussung durch sprachliche Propaganda und eine daraus hervorgehende Anpassung des alltäglichen Sprachgebrauchs an entsprechende schriftliche oder mündliche Quellen. Im Falle entsprechender, islamistischer Denkmuster betreffen diese weniger die sozialen Umstände an sich, sondern eher die geistige, philosophische Haltung, die sich aus diesen ergibt. Man kann bei Erhan A., der im Allgäu lebt und dort nach seinem Abitur ein Wirtschaftsinformatik-Studium begonnen hat, nicht von schwierigen sozialen Umständen sprechen. Auch die Auswanderung seiner Eltern aus der Türkei nach Deutschland kann nicht als traumatische Kindheitserinnerung gewertet werden, wenn man bedenkt, dass er zu diesem Zeitpunkt zwei Jahre alt war (vgl. Delhaes/Obermaier 2014: 14). Soziale Sicherheit bedeutet jedoch nicht, dass die „Notwendigkeit eines persönlichen Positionierens" (Kurbacher 2005: 58) innerhalb der Gesellschaft leichter fällt. Die einfachste Möglichkeit eines solchen Positionierens ist letztlich die Wahl eines vorgegebenen Weges, der klare Strukturen und Halt in Form von Sprach- und Denkmustern bietet - für jenen Weg hat sich Erhan A. letzten Endes entschieden.

\subsection{Sprachorientierte Besonderheiten des Interviews}

Bevor nun konkrete Sprachmuster veranschaulicht werden, soll zunächst kurz das untersuchte Korpus als Ganzes skizziert werden. Im Rahmen der gesamten Analyse ist schließlich zu berücksichtigen, dass es sich dabei nicht um einen primären Propagandatext des Islamischen Staats handelt, der speziell für die Öffentlichkeit angefertigt wurde und die Werte der Terrormiliz dementsprechend ohne Umschweife wiedergibt. Stattdessen wird der Sprachgebrauch eines bekennenden Anhängers im Rahmen eines von zwei Journalisten der Süddeutschen Zeitung durchgeführten Interviews untersucht, d. h., der Interviewte beantwortet spontan - soweit dies anhand der schriftlich fixierten Version eines mündlich durchgeführten Interviews beurteilt werden kann - verschiedene Fragen zu einem übergreifenden Thema, in diesem Falle zu seiner Auffassung seines islamischen Glaubens und der darin begründeten Beziehung zum Islamischen Staat.

Die strukturelle Besonderheit des Interviews im Hinblick auf die zugrunde liegende Hypothese ergibt sich aus der dialogischen Gesprächsform bzw. aus dem Frage-Antwort-Schema, und dessen Konsequenz für den Sprachgebrauch des Interviewten. Die Wortwahl der Antworten muss 
letztlich auch in Anlehnung an die Fragen nachvollzogen werden. Dies bedeutet nicht, dass gesprächsanalytische Aspekte wie der Sprecherwechsel untersucht werden sollen - dies wäre ein ausführliches Thema für sich -, sondern dass die Darstellung sprachlicher Muster anhand eines Interviews zu einem anderen Ergebnis führt als etwa die Untersuchung eines monologischen Textes zur gleichen Thematik. Da der Sprachgebrauch eines Islamisten anhand eines zunächst mündlich durchgeführten Interviews untersucht wird, ist davon auszugehen, dass dieser „ehrlicher" ist, als jener eines schriftlich fixierten Textes, der in der Regel genaue Vorüberlegungen hinsichtlich der Wortwahl impliziert. Dies ist in Hinblick auf die Hypothese insofern interessant, da sich die gedankliche Fixierung des Interviewten in Form von spontanen, sprachlichen Äußerungen nachvollziehen lässt. Auf diese Weise ist der Nachweis einer Fixierung und damit einer bestimmten Meinung allein aufgrund der gegebenen Spontanität besonders aussagekräftig: Genau genommen gibt der Sprecher jene Sprachmuster indirekt wieder, die ihn ursprünglich zu seiner Fixierung bewegt haben. Insofern ergibt sich aus der Textsorte an sich eine Art sprachliche Reflexion.

\section{Methodische Grundlagen}

\subsection{Mittel der Sprachmanipulation nach Olaf Lang}

Um Sprache zugunsten einer als „richtig“ befundenen Überzeugung einzusetzen und widersprüchliche Alternativen auszuklammern, werden bestimmte Mittel eingesetzt, die allesamt das Konzept des Kollektivismus und damit der Vereinfachung gemeinsam haben. Da instrumentalisierte, manipulative Sprache gerade im Rückblick auf die nationalsozialistische Propaganda ein weitreichendes Forschungsfeld der Sprachwissenschaft ist - das aufgrund der Komplexität und notwendigen Interdisziplinarität dennoch keine allgemeingültigen, endgültigen Termini für sämtliche Phänomene zulässt -, stützen wir uns an dieser Stelle auf vorhandenes Material, das insbesondere diejenigen Sprachmuster abdeckt, die im Interview aufgrund der ausreichenden Frequenz eindeutig nachweisbar sind. Aufgrund des direkten Bezugs auf bereits erarbeitete Auffälligkeiten lässt sich letztlich ein ausreichend repräsentativer Wert des Ergebnisses sicherstellen.

Olaf Lang hat im Rahmen der Konferenz Sprachwissenschaft und Sprachkultur bereits 1990 drei charakteristische Merkmale politischer 
Sprachmanipulation ${ }^{6}$ dargelegt, die nun, im Anschluss an die Analyse des Interviews, besonders repräsentativ für radikale Sprachmuster erscheinen. Dabei unterscheidet er zwischen der „sprachliche[n] Verschleierung [...]" (Lang 1991: 53), der „sprachliche[n] Bewertung“(Lang 1991: 54) sowie der Verwendung formelhafter Ausdrücke in Form von Schlagwörtern, Stereotypen und Sprachregelungen. Je nachdem, auf welcher syntaktischen Ebene ein radikaler Kontext schließlich ersichtlich wird, beziehen sich jene Termini auf einzelne Wörter, Wortgruppen, ganze Sätze oder gar Texte. In Bezug auf Schlagwörter, Stereotype und Sprachregelungen ist zu beachten, dass diese nicht zwingend manipulierend und Ausdruck einer radikalen Überzeugung sind, da deren Verwendung oft auf eine ganze Sprachkultur zurückzuführen ist und demnach nicht immer auf eine spezielle Intention verweist (vgl. Lang 1991: 53f.). Ein Beispiel hierfür wäre die stereotype Verwendung von Berufsbezeichnungen: Verwendet man das Wort Lehrer im Plural, um eine kollektive Aussage über jede Person zu treffen, die den Beruf des Lehrers ausübt, kann daraus nicht zwingend auf eine diesbezügliche, radikale Überzeugung geschlossen werden. Von einem radikalen oder manipulativen Gebrauch formelhafter Ausdrücke kann man wohl erst dann sprechen, wenn beim Rezipienten dadurch bewusst negative oder positive Assoziationen hervorgerufen werden sollen.

Um den Befund im Interview nicht auf vorhandene Termini zu reduzieren und somit weitere sprachliche Auffälligkeiten zu vernachlässigen, knüpfen wir an Olaf Langs Einteilung an; diese werden jedoch nicht vollständig übernommen. Stattdessen soll ein schlüssiges Ergebnis erzielt werden, das die Existenz radikaler und extremer Denkmuster des Islamischen Staats aufgrund eines spezifischen, alltäglichen Sprachgebrauchs beweist - insofern müssen all jene Äußerungen des Interviewten erfasst und ausgewertet werden, die sich aufgrund ihrer auffällig hohen Frequenz als wiederkehrendes Schema innerhalb des Interviews abzeichnen. Deshalb erscheint es sinnvoll, Olaf Langs Differenzierung zwischen verhüllten, bewerteten sowie formelhaften Ausdrücken als Anhaltspunkt festzulegen und im Rahmen der Analyse begrifflich anzupassen bzw. zu erweitern. Da sich Letztere zu häufig mit verhüllten und bewerteten Denotaten überschneiden und eher auf politische Reden und Schriften zu beziehen sind,

\footnotetext{
${ }^{6}$ Rückblickend auf die Begriffserklärung des Radikalismus und Extremismus lassen sich diese aus linguistischer Sicht auf bestimmte Formen der politischen Sprachmanipulation zurückführen. Eine radikale bzw. extreme Haltung wird lediglich durch den zuvor erläuterten Vorgang des Überzeugens hervorgerufen, der u. a. als kommunikativer Austausch in Form von sprachlichen Äußerungen erfolgt.
} 
soll resultierend aus der Frequenz des entsprechenden Befundes im Interview stattdessen lediglich auf die Begriffe der Verhüllung und Bewertung eingegangen werden. Während Letzterer selbsterklärend ist und wertende Äußerungen bezeichnet, soll sprachliche Verhüllung kurz anhand eines Beispiels erläutert werden. Als solches nehme man etwa die Aussage Alle anderen sind gegen uns und bezieht sich lediglich auf das Subjekt Alle anderen. Dieses ist nur dann klar definiert, wenn sich aus dem Kontext, in dem dieses verwendet wird, eine außersprachliche Zuordnung durch ein mögliches Ausschlussverfahren ergibt. Dies wäre etwa dann der Fall, wenn mehrere Leute in einem Raum sind und zwei ebenso darin befindliche Personen über Alle anderen sprechen. In allen anderen Fällen, in denen ein solcher Verweis nicht sprachlich geäußert wird, bleibt das Subjekt verschleiert.

Nun ist zu beachten, dass der Sprachgebrauch des Interviewten das Ergebnis einer durch den Vorgang des Überzeugens manipulierten Weltanschauung und damit auch Sprachauffassung ist, d. h., die sprachmanipulativen Mittel wurden unbewusst übernommen. Ziel manipulativer Sprache im politischen Sinne muss es letztlich sein, eine gedankliche Übereinstimmung zwischen Sprecher und Rezipient zu erzeugen, worin die Verbindung zwischen gezielter Sprachmanipulation und radikaler, extremer Sprache an sich begründet ist: Es ist davon auszugehen, dass Letztere in der Regel das Resultat sprachmanipulativer Mittel ist - aus manipulativer Sprache wird somit manipulierte Sprache. Entsprechend der gedanklichen Übereinstimmung muss also auch eine sprachliche Anpassung erfolgen. Es wird also das Resultat einer solchen Anpassung untersucht, nicht die Verwendung der sprachmanipulativen Mittel an sich.

Anschließend an die theoretische Umrahmung entsprechender Sprachmuster lässt sich sagen, dass hinsichtlich der empirischen Analyse des zu untersuchenden Textes diejenigen Äußerungen erfasst und gedeutet werden sollen, die in sprachlicher Form die „Fixierung“ (Kurbacher 2005: $51)^{7}$ einer Überzeugung ausdrücken, d. h., die sich in irgendeiner Weise als Teil eines solchen „Sprachlenkungssystems“ (Schlosser 1995: 139) erweisen und damit die sprachliche Instrumentalisierung des Interviewten

\footnotetext{
7 Kurbacher bezeichnet die „extreme[n] Positionierung“ (Kurbacher 2005: 51) einer Überzeugung als Fixierung. Da zuvor aufgeführt wurde, inwiefern der Ursprung des Radikalismus in einer Überzeugung zu finden ist, bezeichnet der Begriff der Fixierung sowohl deren radikalen als auch extremen Charakter. Im Folgenden soll dieser deshalb weiterhin verwendet werden, um auf die semantische Grundlage der gesuchten Sprachmuster im Allgemeinen zu verweisen.
} 
durch die Machthaber des IS offenlegen. Analog zum Begriff der Fixierung auf philosophischer Ebene könnte man auf sprachwissenschaftlicher Ebene - um die zuvor aufgeführten Mittel zusammenzufassen - von einer sprachliehen Verengung sprechen: Im Fokus der Analyse stehen diejenigen Äußerungen, die im Grunde genommen eine semantische Verengung der Sprache zugunsten einer Fixierung darstellen.

\subsection{Methodische Vielfalt}

Um das Ergebnis jener sprachmanipulativen Mittel anhand entsprechend beeinflusster Sprachmuster methodisch zu erfassen, ist es nicht möglich, sich auf eine einzige Disziplin der Sprachwissenschaft und entsprechende Publikationen zu berufen. Betrachtet man etwa Methoden der Textoder Gesprächslinguistik, die von Klaus Brinker zu einem einheitlichen Konzept zusammengefasst wurden, als Beispiel, wird diese Problematik deutlich. Da das Interview lediglich in schriftlicher Form öffentlich zugänglich ist und als Korpus ausschließlich in dieser Form vorliegt, wäre die Auffassung als Text ${ }^{8}$ und folglich die Verwendung textlinguistischer Methoden sinnvoll, schließlich ist es letzten Endes deren Aufgabe, „Texte in ihre real sozialen Zusammenhänge" (Brinker 2010: 7) einzuordnen. Man könnte sagen, dass jene Zusammenhänge der Erfassung bestimmter Sprachmuster in gewisser Weise zugrunde liegen. Hierbei wäre auch auf das umfassende Textverständnis anderer darauf spezialisierter Linguisten hinzuweisen, so ergibt nach Robert-Alain de Beaugrande und Wolfgang U. Dressler ein Text etwa „nicht von selbst Sinn [...], sondern eher durch die Interaktion von Textwissen mit gespeichertem Weltwissen der jeweiligen Sprachverwender [...]“" (Beaugrande/Dressler 1981: 8). Es bedarf ebenso genau jener Interaktion von Text- und Weltwissen, um sprachliche Strukturen nachzuweisen, welche die zuvor definierte Fixierung eines Sprechers ausdrücken. Dennoch sind die an Lang angelehnten Begriffe letztlich außersprachliche Kategorien, die nicht ausschließlich thematisch oder grammatisch erfasst werden können, da eine entsprechende, umfassende Analyse keine ausreichenden Informationen zum spezifischen Sprachgebrauch des Sprechers hinsichtlich einer einzigen vorausgesetzten Annahme liefern kann. Ein anderes Beispiel zur Veranschaulichung jener Problematik wäre die Analyse nach Sprech-

\footnotetext{
${ }^{8}$ Brinker definiert den Begriff des Textes im linguistischen Sinne als „sprachliche und zugleich kommunikative Einheit [...], d. h. als eine begrenzte, grammatisch und thematisch zusammenhängende (kohärente) Folge von sprachlichen Zeichen, die als solche eine erkennbare kommunikative Funktion (Textfunktion) realisiert" (Brinker 2010: 19f.).
} 
akten, wie sie von Searle 1969 im Rahmen seiner Publikation Speech acts als sprachwissenschaftliches Konzept begründet wurde, wobei die Idee auf John L. Austin zurückzuführen ist. Die „viele[n] Arten von charakteristischen Akten, die mit der Äußerung des Sprechers verbunden sind“ (Searle 2010: 174), könnten ebenso ein Verweis auf die Haltung des Interviewten sein, vorausgesetzt man untersucht dessen Meinungsäußerungen in Form von bestimmten Sprechakten. Jene Analyse könnte die gedankliche Fixierung des Sprechers somit in einem bestimmten, aus Sprechakten bestehenden Muster darstellen, was zunächst ebenso schlüssig erscheint. Dennoch kann auch hierbei, ähnlich wie beim ersten Beispiel, die Individualität des Sprachgebrauchs nicht ausreichend berücksichtigt werden.

Letzten Endes bieten sich zahlreiche Möglichkeiten, um die Äußerungen des Interviewten als Ganzes sprachwissenschaftlich zu untersuchen; es gibt jedoch keine präzise Methode, die einzeln erfasste Ausdrücke eines Sprechers als Phänomene eines radikalen, extremen Weltbildes sprachlich einordnet. Um eine umfassende Theorie solcher Sprachmuster zu entwickeln, wäre die interdisziplinäre Synthese wissenschaftlicher Analysemethoden - etwa aus Bereichen der linguistischen, philosophischen sowie politischen Wissenschaften - denkbar, die den Zusammenhang zwischen sprachlichen Äußerungen und der grundlegenden Idee des Radikalen, bzw. Extremen schematisch darstellen kann.

\subsection{Beschreibung des Analyseverfahrens}

Abschließend zur Klärung der methodischen Vorgehensweise ist erneut darauf hinzuweisen, dass ein weitreichendes Phänomen wie die sprachliche Äußerung islamistischer Denkmuster letztlich kein spezifisches Analyseverfahren einer einzigen, sprachwissenschaftlichen Disziplin zulässt. Nun sollen durch die Analyse zum einen spezifische sprachliche Äußerungen auf die angenommene radikale, extreme Haltung bezogen werden und zum anderen daraus resultierende, wiederkehrende Sprachmuster erfasst werden. Ziel ist also keine vollständige Analyse im Rahmen einer vorhandenen sprachwissenschaftlichen Theorie, sondern der Beweis einer Abzeichnung islamistischer Denkmuster im Sprachgebrauch des Interviewten. Allgemeine stilistische Merkmale - etwa im Rahmen sprachlicher Varietäten wie Umgangs- oder Jugendsprache - sind an dieser Stelle ebenso auszuklammern, da deren Berücksichtigung anstatt der Präzisierung lediglich die Verfälschung des Ergebnisses im Sinne der zu 
beweisenden Hypothese zur Folge hätte. Schließlich kann nicht der allgemeine Sprachduktus des Interviewten als repräsentatives Beispiel für die gesamte Gruppe des IS, die verschiedenste Nationalitäten, Altersklassen etc. einschließt, aufgefasst werden. Um einen allgemeinen, charakteristischen Sprachstil der Gruppe zu erfassen, wäre der sprachwissenschaftliche Bezug auf die arabischen Wurzeln des IS notwendig, wofür die Sprachbarrieren - die ohnehin in jeder Form des sprachlichen Austauschs unbewusst vorhanden sind - letztlich ein zu großes Hindernis darstellen (vgl. Bausinger 1984: 49). Im Fokus der Analyse stehen deshalb lediglich diejenigen sprachlichen Mittel, die sich auf konkrete Denkmuster im Sinne einer Fixierung beziehen und unabhängig von der ursprünglichen Sprache auf die Entstehung einer politisch bzw. religiös motivierten Gruppe zurückzuführen ist.

Um sprachliche Auffälligkeiten bezüglich jener Denkmuster methodisch sinnvoll zu erfassen, soll wie folgt vorgegangen werden: Den an Langs Einteilung angelehnten Kategorien sollen entsprechende Ausdrücke auf Basis von Wörtern bzw. Wortgruppen und syntaktischen Besonderheiten in Hinblick auf die Hypothese zugeordnet werden. Letztlich wird also nach Auffälligkeiten in der Wortwahl gesucht, die auf eine mentale Fixierung des Interviewten hinweisen. Diese schließt einzeln erfassbare Ausdrücke ein, die sich möglicherweise nicht nur als semantisches, sondern auch syntaktisches Muster offenbaren, nicht jedoch den Text als grammatische und thematische Gesamtstruktur - und somit ganze Sätze und Textpassagen, die bezüglich ihrer Aussage interpretiert werden können. Schließlich soll nicht die radikale, extreme Haltung mittels der Analyse allgemeiner Textinhalte, sondern es sollen sprachliche Besonderheiten anhand der bereits vorausgesetzten Haltung nachgewiesen werden. Diese im Rahmen größerer Texteinheiten und damit verbundener Aussagen zu untersuchen, kann zu keinem schlüssigen Ergebnis in Hinblick auf konkrete Sprachmuster führen.

Um diese kategorisch zu ordnen, dient Olaf Langs Einteilung als Anhaltspunkt - der für eine genaue Theorie natürlich noch zu vage, aber dennoch ein erster Ansatz ist, d. h., sprachlich verschleiernde sowie bewertende Äußerungen sollen anhand grammatisch unabhängig deutbarer Ausdrücke mit ausreichend semantischem Gehalt erfasst werden. Die Untergliederung der Kategorien, die später im Rahmen der Beispielanalysen ersichtlich wird, ergibt sich aus der Frequenz im gesamten Interview, die letztlich notwendig ist, um von einem Sprachmuster sprechen zu können. 
Fiktive Feindbilder und verschleierte Gewalt. Sprachwissenschaftliche... 51

\section{Kategorisierte Beispielanalysen}

\subsection{Verschleierung: Fiktive Feindbilder}

5.1.1. Kollektivbezeichnungen durch Substantive, Pronomen und PassivKonstruktionen

Erhan A. bekennt sich im Interview offiziell zum Islamischen Staat und übernimmt damit dessen radikale Weltanschauung, die keine oppositionellen Meinungen bezüglich des gesellschaftlichen Zusammenlebens zulässt. Die daraus resultierende Gegenüberstellung des IS als Instanz des Guten, Gottgewollten und dessen vermeintlichen Widersachern als Instanz des Bösen zeichnet sich im Sprachgebrauch des Interviewten in Form verhüllender Abstrahierungen beider Seiten ab. Diese lassen sich im Interview mittels der vom Interviewten verwendeten Bezeichnungen für das vereinfachte Konstrukt eines „Feindbildes“ bzw. dessen „positives" Gegenbild als wiederkehrendes Schema nachvollziehen. Die sprachliche Äußerung jener konträren Vorstellungen ist zum einen anhand von Ausdrücken erkennbar, die außersprachlich ein Kollektiv an Personen bezeichnen.

$\mathrm{Zu}$ Beginn des Interviews etwa wird Erhan. A. nach der Reaktion seiner Mitschüler auf die strenge Auslegung seines islamischen Glaubens gefragt und referiert in seiner Antwort auf den von den Interviewern verwendeten Ausdruck Ihre Mitschüler (Z. 2)9 ${ }^{9}$, indem er von diesen Andersgläubigen (Z. 4) spricht, wodurch er sich wörtlich von diesen abgrenzt. Im nächsten Satz nimmt er darauf wiederum vergleichend Bezug und verwendet die Muslime (Z. 5) und den darauf verweisenden Ausdruck die anderen Leute (Z. 13), um auf gegnerische Gruppen zu referieren, wobei diese nicht in direkt aufeinanderfolgenden Sätzen verwendet werden, sondern jeweils durch mehrere pronominalisierte Verweise in Form der Personal- und Demonstrativpronomen sie $(\mathrm{Z} .6,10,11,18,19)$ und die $[\text { se }]^{10}(\mathrm{Z} .5,7,10)$ wiederaufgenommen werden. Hierbei ist darauf hinzuweisen, dass er sich selbst aus den bezeichneten Gruppen ausnimmt, indem er von Muslimen (Z. 5) und anderen Leuten [in der Moschee] (Z. 13) spricht und diese mithilfe des bestimmten Artikels

\footnotetext{
${ }^{9}$ Sämtliche Zeilenangaben im Folgenden beziehen sich auf das im Anhang beigefügte SZ-Interview mit Erhan A.

${ }^{10}$ Im Interview verwendet Erhan A. durchgehend den Ausdruck die als pronominalen, umgangssprachlichen Verweis für die 3. Person Plural. Da er zudem sie als Personalpronomen gebraucht, ist davon auszugehen, dass jene Verwendung auf eine verkürzte Form des entsprechenden Demonstrativpronomens diese zurückzuführen ist.
} 
als genaue Personengruppen definiert. Letztlich gehört er - von dem durch Muslime ausgedrückten Denotat ausgehend - selbst der islamischen Religionsgemeinschaft und damit der Gruppe der Muslime an. Zugunsten eines eigens konstruierten Feindbildes jedoch bewirkt er eine sprachliche Gegenüberstellung, die lediglich die Unterscheidung zwischen IS-Anhängern und etwa einem entsprechenden Negativbegriff zulässt, der nicht existent ist. Die auffällig häufige Wiederaufnahme der ohnehin unscharfen substantivischen ${ }^{11}$ Bezeichnungen durch Personal- und Demonstrativpronomen verstärkt deren Undeutlichkeit zusätzlich: Der ursprüngliche Bezugsausdruck verliert letzten Endes zunehmend an Bedeutung und die sprachlichen Grenzen verschwimmen, bis lediglich eine außersprachliche Vorstellung eines gegnerischen Kollektivs bleibt, das aus Sicht des Interviewten als die Anderen bezeichnet werden könnte. Jenes Schema wird fortgeführt, als Erhan A. nach der Reaktion seiner Eltern gefragt wird (Z. 22) und antwortet, die[se] seien wie alle anderen (Z. 23). Die Verwendung von Indefinitpronomen zur Bezeichnung einer bestimmten, nur fiktiv erfassbaren Gruppe von Personen, welcher selbst die eigenen Eltern zugehörig zu sein scheinen, spricht an dieser Stelle für sich. Wer genau jene Gruppe von Anderen ist, kann ebenso nur durch das fiktive Konstrukt eines Feindbildes erfasst werden, das sprachlich jedoch nicht präzisiert wird. Weitere Ausdrücke jenes Feindbildes wären etwa die Amerikaner (Z. 55) als Antwort auf die Frage, wer schuld am Terroranschlag des 11. Septembers sei (Z. 51), oder Feinde (Z. 103) bzw. solche Menschen (Z. 104) zur Bezeichnung der von IS-Soldaten geköpften Journalisten. Seltener, aber ebenso bezeichnend für das entsprechende Schema sind Passiv-Konstruktionen, die ein personenbezogenes Subjekt bzw. Objekt syntaktisch gänzlich aussparen. Ausdrücke wie Wenn auf uns geballert wird (Z. 98) oder ich wurde gezwungen (Z. 184) lassen ebenso auf keinen konkreten außersprachlichen Verweis auf eine oder mehrere Personen schließen.

All jene Bezeichnungen für jegliche Art von Menschen, die - aus Sicht des Sprechers - Teil des negativen Gegenbildes des IS sind, verweisen auf die „Konstruktion eines Gegners, in den alles projiziert wird, was die eigene Aversion, das eigene Ressentiment begründet" (Zehnpfennig 2013: 339). Ein solches Feindbild kann lediglich durch unpräzise Ausdrücke aufrechterhalten werden, welche die Vielfalt der Sprache missachten. Die Anderen bleiben letzten Endes unsichtbar, sowohl in ihrer sprachlichen als auch realen Existenz.

${ }^{11}$ Mit substantivischen Bezeichnungen sind sowohl einzelne Substantive als auch Wortgruppen mit substantivischem Kern gemeint. 
Fiktive Feindbilder und verschleierte Gewalt. Sprachwissenschaftliche... 53

\subsubsection{Verschleierte Gewalt: Modalverben, Indefinitpronomen und} Infinitivkonstruktionen

Erhan A. wird im Interview wiederholt auf die grausamen Methoden des Islamischen Staats angesprochen, der vermeintliche Widersacher weltweit foltert und hinrichtet. Zwar äußert sich Erhan A. unbefangen dazu und spricht von töten (Z. 109), Killen (Z. 224) oder abknall[en] (Z. 204), allerdings niemals mit direktem sprachlichem Bezug auf sich selbst oder verbündete Anhänger. Nach der Erwähnung des Kreuzigens, Steinigens und Köpfens der Gegner des IS in einem eingefügten Kommentar der Interviewer (Z. 96) rechtfertigt Erhan A. die Taten durch den Koran, in dem stehe, dass gläubige Muslime ihre Gegner bekämpfen sollen (Z. 98). Allein das Modalverb sollen rechtfertigt nach seiner sprachlichen Auffassung die Gräueltaten des IS. Jene Kombination aus Modalverb und Verb wird vom Interviewten genau dann gebraucht, wenn die Brutalität und Skrupellosigkeit der Gruppe sprachlich zum Ausdruck gebracht wird. Ein weiteres Beispiel wäre etwa [Christen und Fuden] können [...] weiterleben (Z. 119). Indem also die Opfer des IS zum sprachlichen Subjekt werden und der Agens (Täter) gänzlich fehlt, wird der direkte Bezug auf den Islamischen Staat als Ausgangspunkt der Gewalt vermieden - der Ausdruck weiterleben impliziert die Gewalt an dieser Stelle nur indirekt.

Zusätzlich werden jene Verben entweder in Verbindung mit man als Indefinitpronomen oder gar als Infinitivkonstruktionen gebraucht, wodurch die gleiche Wirkung erzielt wird. Ausdrücke wie [Kriegsgefangene] [...] darf man töten (Z. 109), Man kämpft (Z. 138) und [legitim,] sie zu töten (Z. 199) zeichnen sich ebenso durch ihr verschleiertes bzw. nicht vorhandenes Subjekt aus und werden vom Sprecher ohne persönlichen Bezug wiedergegeben. Mit jenem fehlenden Selbstbezug streitet Erhan A. jegliche Verantwortung des IS ab und bedient sich der „Handlungsanleitung zur Tötung“ (Sailer-Wlaists 2012: 12), die er als Teil des „Bedeutungsraum[es] des Gottgewollten“ (Sailer-Wlaists 2012: 12) auffasst und sprachlich dementsprechend nicht rechtfertigen kann.

Die einzige diesbezügliche Ausnahme hat im Hinblick auf das aufgeführte Schema eine besondere Stellung im gesamten Interview. Nur ein einziges Mal bekennt sich Erhan A. sprachlich konkret zu seiner Gewaltbereitschaft, indem er zugibt Ich würde [sogar meine Familie] töten (Z. 122). Hiermit drückt er mit genauem Verweis auf sich selbst durch das Personalpronomen - trotz des Konjunktivs - seine offene Bereitschaft zu töten 
aus. Betrachtet man jene Besonderheit im Hinblick auf die rechtlichen Konsequenzen des Interviews, nämlich die Ausweisung des Islamisten in die Türkei, ist dies möglicherweise die Schlüsselstelle des Interviews, auf die sich die Rechtsprechung berufen kann.

\subsection{Bewertung: Adverbiale, adjektivische und präpositionale Polarisierung}

Bezüglich seiner Äußerungen zum eigenen Glauben, dem Islam, operiert Erhan A. sprachlich beinahe ausnahmslos mit Wertungen, die in Form von Prädikativergänzungen oder innerhalb substantivischer oder adjektivischer Wortgruppen auftreten. Erstere beziehen sich dabei allesamt auf die Ausübung des islamischen Glaubens, welche er damit in zwei Kategorien - vereinfacht ausgedrückt in eine richtige und falsche Ausübung - einteilt. Beispiele hierfür sind praktizieren [den Islam] nicht richtig (Z. 18), beten falsch (Z. 18) oder islamisch korrekt [sein] (Z. 40f.). Letztere verweisen wiederum direkt auf den Islam bzw. dessen idealisierte Form eines Islamischen Staats, der somit den gleichen, sich gegenseitig ausschließenden Kategorien zugeordnet wird. So spricht Erhan A. vom falsche[n] Verständnis [vom Islam] (Z. 27), dem Islam als einzig wahre Religion oder einem echten islamischen Staat (Z. 89). Nicht objektivierbare Phänomene wie Religion und Glaube werden auf diese Weise in überschaubare Kategorien wie richtig oder falsch eingeordnet, wodurch deren begriffliche, gedankliche Vielfalt sprachlich verfehlt wird.

Zusätzlich zu jenen adverbialen bzw. adjektivisch geäußerten Wertungen werden die Präpositionen für und gegen gebraucht, um das Feindbild und das vorausgesetzte positive Gegenbild zu polarisieren. Durch für [die] (Z. 41) drückt Erhan A. das Zugehörigkeitsgefühl zu islamisch korrekt[en] (Z. 40) Gruppen aus, während er durch gegen [mich] (Z. 23) eine angenommene gegnerische Haltung alle[r] anderen (Z. 23) impliziert.

\subsection{Semantische Einzelfälle}

Zuletzt ist auf zwei Bezeichnungen zu verweisen, die nicht als wiederkehrendes sprachliches Muster zu erfassen sind, aber in ihrer einzelnen Bedeutung besonders aussagekräftig bezüglich der radikalen, extremen Haltung des Interviewten sind. Dies ist zum einen der neologistische Ausdruck Euro-Fake-Islam (Z. 89) als Bezeichnung für die Praktizierung des islamischen Glaubens in der Türkei. Das negativ konnotierte Wort entpuppt sich ebenso als sprachlicher Ausdruck des kons- 
Fiktive Feindbilder und verschleierte Gewalt. Sprachwissenschaftliche... 55

truierten Feindbildes, wobei hierbei konkret auf Europa und damit den Westen Bezug genommen wird.

Ebenso aufschlussreich ist die semantische Verschiebung des negativ konnotierten Wortes Terroristen (Z. 46) im Rahmen des vom Sprecher hergestellten gedanklichen Kontextes. Erhan A. teilt an dieser Stelle seine verbündete Haltung zur Terrormiliz Al Qaida mit und behauptet, er hätte diese nur in der Vergangenheit für Terroristen (Z. 46) gehalten. Da er die Frage, ob Al-Qaida-Anhänger denn keine Terroristen seien, verneint, ist davon auszugehen, dass er die eigentliche Bedeutung des Wortes Terrorist zugunsten seines Weltbildes gedanklich verzerrt.

\section{Die sprachliche Vereinfachung einer Weltanschauung}

Sämtliche Äußerungen, die im Rahmen der Untersuchung erfasst wurden, haben eine Funktion gemeinsam: Sie stellen alle in bestimmter Weise die sprachliche „Reduktion der Komplexität“ (Sailer-Wlaists 2012: 16) verschiedener Weltauffassungen dar. Anstatt auf die Vielfalt an Möglichkeiten, die Sprache zur Erfassung dieser bietet, zurückzugreifen, bedient sich Erhan A. festgefrorener Strukturen, die einer gedanklichen Fixierung entsprechen. Mithilfe sprachlich konstruierter aufgezeigter Muster vermeidet er kritische Differenzierungen und entzieht sich damit seiner „auferlegten Freiheit zur Verantwortung“ (Kurbacher 2005: 56).

\section{Literaturverzeichnis}

Bausinger, Hermann (1984): Deutsch für Deutsche. Dialekte, Sprachbarrieren, Sondersprachen. Frankfurt am Main.

Brinker, Klaus (2010): Linguistische Textanalyse. Eine Einführung in Grundbegriffe und Methoden. 7., durchgesehene Auflage. Berlin.

Bötticher, Astrid/Mareš, Miroslav (2012): Extremismus. Theorien, Konzepte, Formen. München.

De Beaugrande, Robert/Dressler, Wolfgang (1981): Einführung in die Textlinguistik. Tübingen.

De Saussure, Ferdinand (1967): Grundfragen der allgemeinen Sprachwissenschaft. 2. Auflage. Berlin.

Fuest, Leonhard/Löffler, Jörg (Hg.) (2005): Diskurse des Extremen. Über Extremismus und Radikalität in Theorie, Literatur und Medien. Würzburg.

Kurbacher, Frauke Annegret (2005): Radikalität als Denkfigur. Zur Philosophie extremer Überzeugungen. In: Fuest, Leonhard/Löffler, Jörg (Hg.): Diskurse des Extremen. Über Extremismus und Radikalität in Theorie, Literatur und Medien. Würzburg, S. 49-60. 
Lang, Olaf (1991): Sprachkultur und politische Kommunikation. In: Sommerfeldt, Karl-Ernst (Hg.): Sprachwissenschaft und Sprachkultur. Tagungsband der Konferenz in Neubrandenburg am 10. und 11. Mai. Frankfurt am Main, S. 51-58.

Ryborz, Heinz (2012): Beeinflussen, Überzeugen, Manipulieren. Seriöse und skrupellose Rhetorik. Regensburg.

Said, Benham T. (2014): Islamischer Staat. IS-Miliz, al-Quaida und die deutschen Brigaden. München.

Sailer-Wlasits, Paul (2012): Verbalradikalismus. Kritische Geistesgeschichte eines soziopolizisch-sprachphilosophischen Phänomens. Wien.

Schlosser, Horst Dieter (1995): Die Sprache von Extremismus und Gewalttätigkeit. In: Zwiener, Ulrich et al. (Hg.): Gegen Extremismus und Gewalt. Jena, S. 137-167.

Searle, John R. (2010): Was ist ein Sprechakt? In: Hoffmann, Ludger (Hg.): Sprachwissenschaft. Ein Reader. 3. Auflage. Berlin, S. 174-193.

Willer, Stefan (2005): Radikalität als Sprachspiel. In: Fuest, Leonhard/Löffler, Jörg (Hg.): Diskurse des Extremen. Über Extremismus und Radikalität in Theorie, Literatur und Medien. Würzburg, S. 61-73.

Zehnpfennig, Barbara (2013): Das Weltbild von Sayyid Qutb - Der Dschihad als Verwirklichung des richtigen Lebens. In: Hirscher, Gerhard/Jesse, Eckhard (Hg.): Extremismus in Deutschland. Schwerpunkte, Vergleiche, Perspektiven. Baden-Baden, S. 327-348.

\section{Quellen}

Delhaes, Marie/Obermaier, Frederik (2014): Ich glaub, das steht irgendwo im Koran. In: Süddeutsche Zeitung Magazin Nr. 40 (2014), S. 13-19.

\section{Digitales Wörterbuch}

http://www.dwds.de (12.02.2019).

\section{Fikcyjny wróg i zawoalowana przemoc. Językoznawcza analiza wywiadu z islamistą}

Abstrakt: Przedmiotem rozważań artykułu jest wywiad dwójki dziennikarzy z Erhanem A., „wyznawcą“ IS, który ukazał się w Süddeutsche Zeitung (nr 40, 2014, 13-19). Celem publikacji jest wyizolowanie islamistycznych wzorców myślowych poprzez analizę specyfiki doboru słownictwa. Czynnik powtarzalności („wzorce językowe“) w przytaczanych przykładach jest przy tym decydujący dla 
Fiktive Feindbilder und verschleierte Gewalt. Sprachwissenschaftliche.. 57

doboru kryteriów analizy. Pod uwagę zostały także wzięte aspekty wynikające ze struktury wywiadu. Analiza koncentruje się na werbalizowaniu nazw kolektywnych oraz zawoalowanej przemocy. Wyizolowana (semantyczna) nieostrość w wyrażeniach powstaje między innymi poprzez zastosowanie konstrukcji strony biernej, jak również zaimka nieokreślonego. W szerszym ujęciu niniejszy artykuł stanowi językoznawcze badanie poświęcone użyciu w islamie języka niemieckiego, w węższym zaś - języka niemieckiego przez zwolenników tak zwanego Państwa Islamskiego (IS).

Słowa kluczowe: wywiad, „wyznawca“ IS, „wzorce językowe“, nazwy kolektywne, zawoalowana przemoc.

\title{
Fictitious Enemy Images and Veiled Violence. Linguistic Analyses of an Interview with an Islamist
}

\begin{abstract}
This article examines an interview of two journalists with Erhan A., a confessor of the IS, which appeared in the magazine of the Süddeutsche Zeitung (No. 40, 2014, 13-19). The aim is to bring out Islamist thought patterns through conspicuous features in the choice of words. The moment of repetition ("language patterns") in the examples is decisive for the selection of the analysis categories. Special features which result from the interview structure are taken into account. The focus of the analysis is on the verbalization of collective names and veiled violence. The identified (semantic) vagueness results from passive constructions and, for example, from indefinite pronouns. In a superior sense, it is a linguistic work on the German language used in Islam or in the narrower sense on the use of the German language in statements by advocates of the so-called Islamic State (IS).
\end{abstract}

Key words: Interview, confessor of the IS, "language patterns", verbalization of collective names and veiled violence. 



\title{
ANHANG
}

\section{Das folgende Interview stammt aus dem SZ-Magazin vom 2. Oktober 2014. Der genaue Verweis auf die Primärquelle ist dem Literaturverzeich- nis zu entnehmen. Kommentierende Zusätze der Interviewer wurden nicht übernommen und sind entsprechend markiert.}

\author{
$[\ldots]$
}

Sie wurden von heute auf morgen ein strenggläubiger Muslim, einer, der den Koran wörtlich auslegt. Wie haben Ihre Mitschüler reagiert?

Die haben nur einmal gefragt, warum ich das mache. Ich habe ihnen vom Koran erzählt. Damit war die Sache erledigt. Von diesen Andersgläubigen hätte ich mehr Kritik erwartet. Aber die waren toleranter als die Muslime. Aus allein drei Moscheegemeinden in Kempten haben sie mich und meine Freunde rausgeschmissen. Die wollten uns nicht dort haben.

\section{Warum nicht?}

Sie meinten, wir seien Wahhabiten, Salafisten, Radikale. Die hatten auch was gegen meinen Bart. Sie meinten, dass ich ihn kürzen soll. Ich habe dann gesagt: Nein, der Bart ist Pflicht, so steht es im Koran. Damit fing das Ganze an. Wir haben ständig diskutiert. Wir haben die anderen Leute in der Moschee auf ihre Fehler hingewiesen. Aber sie wollten es nicht verstehen. Das war uns auch egal. Im Koran steht, dass man Gegenwehr bekommt. Das hat uns gerade bestätigt, dass es richtig ist, was wir tun.

Auf was für Fehler?

So Kleinigkeiten. Sie praktizieren den Islam einfach nicht richtig, sie beten falsch, und außerdem lehnen sie die Demokratie nicht ab. Demokratie und Islam, das ist wie Feuer und Wasser. Es ist nicht miteinander vereinbar.

Was haben Ihre Eltern gesagt?

Die sind wie alle anderen, die sind gegen mich.

Hat Ihnen das nicht zu denken gegeben? Ihre Eltern sind immerhin auch Muslime. Mein Vater nicht. Der betet nicht mal. Meine Mutter betet noch, aber sie hat ein falsches Verständnis vom Islam. Sie versucht, die Wörter im Koran umzudrehen und umzudeuten. Ich mache genau das, was im Koran steht, und nur das ist richtig, Punkt.

$[\ldots]$

Nun ist es ja ein Unterschied, ob man streng nach dem Koran lebt oder ob man nach Syrien geht, um sich einer islamistischen Gruppe anzuschließen.

Ja, das wollten wir am Anfang auch überhaupt nicht. Wir haben das nur beobachtet. Wir haben uns Nachrichten angeschaut, ARD und ZDF und so, aber uns war schnell klar: Da wird nur Scheiße berichtet. Da haben wir uns dann im Internet Videos angeguckt.

Welche Videos?

Von Leuten, die dort sind. Wir haben geschaut, welche Gruppierungen islamisch korrekt sind, welche der islamischen Ideologie entsprechen, und für die waren wir dann.

Für den Islamischen Staat?

Ja. Und für Al-Qaida. Ich habe früher schlecht über Al-Qaida gedacht. Ich habe auch gedacht: Das sind Terroristen. 
Wer war es dann?

Es waren die Amerikaner selbst.

Wann haben Sie das nächste Mal von David G. gehört?

Wir hatten uns verabschiedet, und eine Woche danach bekam ich einen Anruf. Von einer türkischen Nummer. Ich wollte erst gar nicht rangehen, hab es dann aber doch gemacht. David war dran. Da fragte ich: Hey, cool, wie hast du denn das gemacht? Er so: Egal.

Ein paar Tage später ist er über die Grenze nach Syrien gelangt. Wie sind Sie in Kontakt geblieben?

Übers Internet, Facebook und so.

65

Hatten Sie das Gefühl, dass es ihm gut geht?

Ja, klar. Man lebt da unten gut. Die haben einfach alles: Laptops, Waffen, Knabberzeugs. Voll der Luxus. Ich habe ja die Bilder von David gesehen, wie er mit anderen chillt.

Auf einem dieser Bilder trägt er ein T-Shirt von Al-Qaida. Einige Zeitungen haben es gedruckt.

Ja, ich fand das voll cool. Ganz Kempten hat darüber geredet, eigentlich ganz Deutschland. So was hatte sich ja vorher niemand getraut. Ich habe das Bild gesehen und mir gedacht: Der steht echt dazu.

Wie oft haben Sie mit David G. gesprochen?

Einmal die Woche haben wir geschrieben. Irgendwann hab ich ihm dann gesagt, dass ich auch komme. Und er so: Ey, cool.

Es vergeht kein Tag ohne Horrornachrichten aus dem Irak und Syrien. Die Welt hat Angst. Ausgerechnet dieser Gruppe wollten Sie sich anschließen. Warum? Der Islam ist die einzig wahre Religion. Weltweit haben wir leider keinen einzigen echten islamischen Staat. Nur Staaten wie die Türkei, wo so ein Euro-Fake-Islam gelebt wird. IS will aber einen echten islamischen Staat, einen, wo der Koran auch so gelebt wird, wie es Allah will. Und IS ist auf dem besten Weg, das auch zu schaffen.

Mit brutalsten Mitteln.

Ich weiß nicht, wo da die Brutalität wäre.

Leute, die sich dem IS nicht beugen, werden gekreuzigt, gesteinigt und geköpft. Ich befürworte nicht alles, was die Gruppe macht. Aber im Koran steht nun mal, dass wir diejenigen bekämpfen sollen, die uns bekämpfen. Wenn auf uns geballert wird, dann können wir ja nicht einfach dastehen und sagen: $\$$ Ja, macht es doch halt! « Wir müssen 
Fiktive Feindbilder und verschleierte Gewalt. Sprachwissenschaftliche... 61

uns wehren. Wenn man für eine gute Sache tötet, ist das legitim.

Ist es in Ihren Augen auch legitim, Journalisten zu köpfen?

Ich habe gehört, das waren Spione. Also Feinde. Und die darf man töten. Wenn Allah sagt, es ist erlaubt, solche Menschen zu töten, dann würde ich das auch machen. Ich folge seinen Gesetzen blind.

Würden Sie auch gefesselte Männer erschießen? Auf Dutzenden IS-Videos im Internet ist genau das zu sehen.

Das waren Kriegsgefangene, die darf man töten, die haben auf unsere Leute geschossen.

Und was ist mit Zivilisten?

Denen passiert nichts. Unschuldige zu töten ist gegen den Islam.

IS hat auf seinem Vormarsch schon Hunderte Menschen getötet. Wie passt das zusammen?

Ich kann nicht für die ganze Gruppe sprechen. Es gibt sicher immer wieder Einzelne, die Fehler machen. Aber wenn sich Zivilisten dem Islamischen Staat beugen, wird ihnen nichts passieren. Auch Christen und Juden können übrigens weiterleben. Sie müssen sich halt an die islamischen Gesetze halten und Steuern zahlen, quasi ein Schutzgeld. Wenn sie dazu aber nicht bereit sind, dann werden sie auch getötet. Ich würde sogar meine Familie töten, wenn sie sich gegen den Islamischen Staat stellt.

\section{[...]}

Als Sie in der Türkei waren, starb Ihr Freund David. Wie haben Sie von seinem Tod erfahren?

Ein gemeinsamer Freund, der auch in Syrien ist, hat es mir geschrieben.

Waren Sie traurig?

Am Anfang. Aber ich habe ihn auch ein bisschen beneidet.

Warum?

Ja, ich weiß ja, wo er jetzt ist.

Sie meinen, dass er sich jetzt im Paradies mit 72 Jungfrauen vergnügt und vor Bächen voll Wein sitzt?

Ach, die Frauen und der Wein, das sind nur kleine Details. Man kämpft aber doch nicht für ein paar Frauen, darum geht es nicht. Damit wird das Ganze immer lächerlich gemacht. Dann heißt es wieder, wir sprengen uns für ein paar Frauen in die Luft. Um ehrlich zu sein: Wenn es nur die Frauen gäbe, würde ich es nicht machen. Ich mache es für Allah.

Wissen Sie, wie Ihr Freund David gestorben ist?

Er starb im Kampf, in der Nähe von Aleppo. Er wurde in die Schulter geschossen. Er ließ sich verarzten und hat gleich weitergekämpft. Dann wurde er in die Hand geschossen. Er ließ sich wieder verarzten und machte weiter. Dann wurde er noch mal getroffen und ist halt irgendwann zu Boden gegangen. 
Hat Sie der Anblick von Davids Leiche schockiert?

Nein. Mir war ja von Anfang an klar, dass er nicht mehr zurückkommt. Nachdem ich gesehen habe, was mit ihm passiert ist, wollte ich erst recht gehen. Das hat mir so einen richtigen Schub gegeben.

Haben Sie Ihrer Familie erzählt, was Sie vorhaben?

Nein, nicht einmal meine Freunde hier in Kempten wussten was. Meine Eltern haben es aber wohl geahnt. Sie haben mir Hunderte Nachrichten geschrieben und versucht, mich anzurufen. Ich habe nicht reagiert. Aber dann haben sie meinen Onkel in der Türkei angerufen, bei dem ich damals untergekommen bin.

\section{Wie hat er reagiert?}

Er wollte mich aufhalten. Aber irgendwann hat er aufgegeben.

Wie haben Sie den Kontakt zu den Islamisten in Syrien hergestellt?

Ich hatte Telefonnummern von David bekommen. Die musste ich nur anrufen, und dann haben die das klargemacht.

Das hört sich an wie ein Reisebüro.

So in etwa. Sie haben mir gesagt, dass ich in die Stadt Hatay fahren soll, und da würden dann schon Leute warten, die mich über die Grenze bringen.

Hatten Sie keine Bedenken, dass es sich um eine Falle handelt? Ich kannte die Leute ja, mit denen ich telefoniert habe.

Waren das Leute aus Deutschland?

Teilweise.

Aus Dinslaken?

Dazu sage ich jetzt nichts.

Jetzt sitzen Sie aber hier in Kempten, nicht in Aleppo. Was ist passiert?

In Hatay musste ich nur noch in den Bus steigen. An der Bushaltestelle waren dann aber plötzlich überall Polizisten. Da dachte ich mir: Okay, wenn ich da jetzt nicht einsteige, dann können sie mir auch nichts anhaben. Und dann bin ich halt wieder zurückgegangen zu meinem Onkel. Ich wurde gezwungen, mir meinen Bart abzuschneiden. Alle meinten, damit sei ich entradikalisiert. Aber insgeheim habe ich mir geschworen, dass ich noch krasser werde.

\section{$[\ldots]$}

Ein Dinslakener wurde bekannt, weil er auf einem Foto mit mehreren abgeschlagenen Köpfen posiert und in die Kamera gegrinst hat. Kennen Sie ihn?

Ja, das ist Mustafa. Ich chatte regelmäßig mit ihm. Der ist sehr nett, hat eine angenehme Stimme.

Wissen Sie, ob er es war, der die Männer geköpft hat?

Das weiß ich nicht. Aber ich denk mal, dass er es war. 
Fiktive Feindbilder und verschleierte Gewalt. Sprachwissenschaftliche... 63

\section{Finden Sie das gut?}

Ja. Das waren Assad-Leute, es war also legitim, sie zu töten. Ob man dann auch noch mit den Köpfen rumposen muss, das ist wieder eine andere Sache. Das hätte ich nicht gemacht.

Warum schneiden die Kämpfer des IS ihren Gegnern die Köpfe ab?

Ich glaub, das steht irgendwo im Koran. Und ob man die Feinde abknallt oder köpft, ist doch egal. Tot ist tot. Es geht nur darum, dass sie nicht leiden. Selbst die schlimmsten Feinde dürfen wir nicht quälen, so will es der Koran.

Ein anderer Mann aus Dinslaken hat sich im Juli bei einem Selbstmordanschlag in die Luft gesprengt.

205 Ja, das war Philipp, mit dem hab ich auch oft telefoniert. Es wird erzählt, dass er sich freiwillig gemeldet hat, weil er eh schon verletzt war und nicht mehr reden konnte. Aber ich denke, dass er einfach den Islam voranbringen wollte und sich für Allah geopfert hat. Er hätte sich sicher auch bereit erklärt, wenn er nicht angeschossen gewesen wäre.

Würden Sie so was auch machen?

Nein, das würde ich mich nicht trauen.

\section{[...]}

IS kontrolliert mittlerweile große Teile Syriens und des Irak. Glauben Sie, dass der Vormarsch noch weitergeht?

Als Nächstes kommt hoffentlich die Türkei. Wenn die Türken sich nicht widersetzen, wird die Scharia eingeführt. Ohne Stress und ohne Killen. Und dann geht es weiter ins nächste Land.

Auch Deutschland?

Ja, klar. In zwanzig, dreißig Jahren haben wir das geschafft. Wir kämpfen so lange, bis der ganze Planet islamisch ist.

Und dann?

Erst mal wird der Islam zur Staatsreligion gemacht. Dann kommt überall unsere Flagge hin. Und die Polizeiautos machen wir schwarz statt grün. Sonst würde alles so bleiben. Christen und Juden dürfen ja ihre Religion hier weiterleben. Sie müssten halt eine Steuer zahlen. Und natürlich müssen sie sich an unsere Gesetze halten. Frauen ohne Schleier, das ist zum Beispiel ein No-Go.

Einen Tag nach Ihrer Rückkehr aus der Türkei nach Deutschland, nach Ihrem missglückten Einreiseversuch nach Syrien, stand die Polizei vor Ihrer Tür. Was wollten die Beamten?

Die haben meinen Pass einkassiert. Seitdem muss ich mich zweimal pro Woche bei der Polizei melden. Vor ein paar Wochen habe ich dann auch noch einen Platzverweis für die Allgäuer Festwoche bekommen, die hatten Angst, dass ich ein Attentat begehe. Die Festwoche ist ein Volksfest, so eine Art kleines Oktoberfest. Mit Musik, Frauen und Alkohol. Das widert mich an. Aber ein Attentat? Das hätte ich echt nicht gemacht. 
Haben Sie noch Kontakt zu Ihren Freunden in Syrien?

Ja klar, wir haben eine Whats-App-Gruppe, Dawla al-Islamiya, also Islamischer Staat, heißt die. Da sind ungefähr 50 Leute drin. Etwa ein Drittel ist schon in Syrien oder dem Irak, der Rest überlegt, bald nachzukommen.

245

Worüber unterhalten Sie sich?

Über alles, was zum Islam gehört. Die Leute, die schon angekommen sind, erzählen halt, was so passiert. Wo zum Beispiel eine Bombe eingeschlagen ist, wer gestorben ist.

Unterhalten Sie sich auch darüber, wer als Nächstes nach Syrien geht? Nein, darüber sprechen wir aus Sicherheitsgründen nicht.

Politiker und Experten rätseln, wie man deutsche Islamisten davon abhalten könnte, nach Syrien zu gehen. Wie könnte man Sie aufhalten?

Das können sie nicht. Das haben die Behörden jetzt auch eingesehen. Meinen Pass jedenfalls habe ich vor ein paar Tagen wieder zurückbekommen. Jetzt werde ich erst mal für ein paar Wochen in den Urlaub fahren.

Wirklich nur in den Urlaub? Oder doch nach Syrien? Mal schauen. 